\title{
Direct peroral cholangioscopy using an ultrathin endoscope: Making technique easier
}

\author{
Javier Sola-Vera, Francisco Uceda, Rubén Cuesta and Narcís Vázquez
}

Digestive Endoscopy Unit. Section of Digestive Diseases. Hospital General Universitario de Elche. Elche, Alicante. Spain

\begin{abstract}
Background: Cholangioscopy is a useful tool for the study and treatment of biliary pathology. Ultrathin upper endoscopes allow direct peroral cholangioscopy (DPC) but have some drawbacks.

Objective: The aim of the study was to evaluate the success rate of DPC with an ultrathin endoscope using a balloon catheter to reach the biliary confluence.

Material and methods: Prospective observational study. An ultrathin endoscope (Olympus XP180N, outer diameter $5.5 \mathrm{~mm}$, working channel $2 \mathrm{~mm}$ ) was used. To access the biliary tree, free-hand technique was used. To reach the biliary confluence an intraductal balloon catheter (Olympus B5-2Q diameter $1.9 \mathrm{~mm}$ ) and a 0.025 inch guide wire was used. In all cases sphincterotomy and/ or sphincteroplasty was performed. The success rate was defined as the percentage of cases in which the biliary confluence could be reached with the ultrathin endoscope.
\end{abstract}

Results: Fifteen patients ( 8 men/7 women) were included. Mean age was $77.7 \pm 10.8$ years (range 45-91). The indications for cholangioscopy were suspected bile duct stones $(n=9)$, electrohydraulic lithotripsy for the treatment of difficult choledocholithiasis $(n=5)$ and evaluation of biliary stricture $(n=1)$. Access to the bile duct was achieved in 14/15 cases (93.3\%). Biliary confluence was reached in $13 / 15$ cases (86.7 \%). One complication was observed in one patient (oxigen desaturation).

Conclusions: DPC with an ultrathin endoscope can be done with the free-hand technique. Intraductal balloon-guided DPC allows full examination of the common bile duct in most cases.

Key words: Direct peroral cholangioscopy. Ultrathin upper endoscope. Endoscopic retrograde cholangiopancreatography.

Received: 02-08-2013

Accepted: 09-01-2014

Correspondence: Javier Sola-Vera Sánchez. Section of Digestive Medicine. Hospital General Universitario de Elche. Camí de l'Almazara, 11. 03203 Elche, Alicante. Spain

e-mail: solavera_jav@gva.es
Sola-Vera J, Uceda F, Cuesta R, Vázquez N. Direct peroral cholangioscopy using an ultrathin endoscope: Making technique easier. Rev Esp Enferm Dig 2014;106:30-36.

\section{INTRODUCTION}

Cholangioscopy is a useful technique for the study and treatment of biliary diseases (1). Systems available today for peroral cholangioscopy have some limitations. The "mother-baby" systems are fragile and require the presence of two skilled endoscopists. The SpyGlass ${ }^{\circledR}(2)$ and Polyscope $^{\circledR}$ (3) allow performance of single-operator cholangioscopy, being its drawbacks that the image quality is not optimal and the working channel has a small diameter.

Ultrathin endoscopes are increasingly available in endoscopy units and may be useful for the performance of direct peroral cholangioscopy (DPC) (4). They present several advantages: They can be handled by a single operator, provide high resolution digital images and have a working channel which is larger than the above mentioned cholangioscopy systems. However, DPC with ultrathin endoscope has important limitations. Access to the biliary tract has been previously described as cumbersome, time consuming and associated with high failure rate. Even when access is achieved, it is difficult to advance the ultrathin endoscope to the biliary confluence, due to the formation of loops in the stomach and duodenum. To solve this problem several systems have been investigated such as overtubes (5), the use of intraduodenal balloon (6), intraductal anchoring systems with special guide wires (7) and intraductal balloon-assisted cholangioscopy (8).

The aim of the study was to evaluate the success rate to reach the biliary confluence using an ultrathin endoscope with an intraductal balloon. 


\section{MATERIAL AND METHODS}

A prospective study was conducted. Consecutive patients with biliary pathology were enrolled in this study between December 2012 and June 2013. Exclusion criteria were: Age under 18 years, any type of gastric surgery or surgery on the biliary tract, coagulopathy, acute cholangitis, biliary sepsis and bile duct diameter less than $6 \mathrm{~mm}$. The study was conducted in the setting of a university hospital.

The primary endpoint was to successfully achieve a complete examination of the biliary tree, which was defined as the percentage of cases in which the examination of the common bile duct to the confluence was possible. The secondary endpoint was to successfully access the main bile duct with the free-hand technique, i.e., without the need of a guidewire to insert the endoscope, defined as the percentage of cases in which the tip of the ultrathin endoscope passed through the papilla clearly standing within the bile duct.

Demographics data as well as the indication for cholangioscopy and diameter of the common bile duct were collected. The access time was defined as the time between insertion of the ultrathin endoscope through the mouth until its tip was situated anywhere in the lower third of the bile duct. The time to confluence was defined as the time from first visualization of the bile duct until the tip of the endoscope reached a distance of $1 \mathrm{~cm}$ or less from the biliary confluence.

The total time was calculated from the moment the ERCP began, including cholangioscopy, until the entire procedure ended. The percentage of cases in which it was possible to examine the bile duct confluence was calculated by intention-to-treat analysis. Complications were recorded in the first 24 hours after the procedure, while the patient remained in the hospital. No systematic complementary examinations were performed in patients after the procedure, unless clinically indicated. Complications after discharge were recorded by reviewing the medical record of each patient 30 days after the procedure

In all patients, endoscopic retrograde cholangiopancreatography (ERCP) was done as usual. Briefly, the patient was placed in the left lateral position and put into deep sedation by an anaesthesiologist. In the "naive" papilla an indomethacin suppository was administered just before the procedure. Bus Copan ${ }^{\circledast}$ was not systematically administered. Antibiotics were administered i.v. before and after ERCP overnight. The examination was performed with $\mathrm{CO}_{2}$ insuflation. Olympus TJF-160VR and TJF- Q180V duodenoscopes were used. Fluoroscopic guidance with mobile C-arm (BV Endura, Philips $^{\circledR}$ ) was used. Papilla cannulation was performed with sphincterotome over a 0.025 inch guidewire. After the diagnostic cholangiography, sphincterotomy and/or balloon sphincteroplasty were performed in the "naive" papillae, as needed to address the pathology of the bili- ary tract. The calibre of sphincterotomy and/or sphincteroplasty was given by the underlying pathology and the expected therapeutic manoeuvres. In patients in whom a previous sphincterotomy and/or sphincteroplasty had been done, the papilla was dilated only if the size of the orifice was judged to be insufficient for the expected therapeutic manoeuvres.

\section{DPC technique}

After removing the duodenoscope, an ultrathin endoscope (Olympus ${ }^{\circledR}$ XP180N with outer diameter of $5.5 \mathrm{~mm}$ and working channel of $2 \mathrm{~mm}$ ) was introduced through the mouth into the duodenum. To access the biliary tree, we attempted to advance the endoscope through the papilla into the common bile duct, pushing the endoscope or performing a "J" manoeuvre, i.e., bending the tip of the endoscope in order to achieve a retroflexion position after which the endoscope was withdrawn and contact the papilla.

To access the common bile duct, sometimes it was useful a counter-clockwise rotation of the endoscope. Once the lower third of the bile duct was visualized, a lubricated Fogarty balloon (Olympus ${ }^{\circledR}$ B5-2Q, external diameter $1.9 \mathrm{~mm}$ ) was passed through the working channel of the endoscope. This balloon has exactly the same composition as the standard Fogarty balloons used in ERCP, containing no metal parts.

A standard guide wire (VisiglideTM 0.025 inch, Olympus ${ }^{\circledR}$ ) was used to guide the balloon. After advancing the balloon catheter into a branch of an intrahepatic duct via the guidewire, the balloon was inflated to $11 \mathrm{~mm}$ to anchor it within the duct. Under fluoroscopic and endoscopic control, the endoscope was carefully advanced over the balloon catheter. During this manoeuvre the balloon could slip from the biliary tree necessitating its reposition to a more proximal branch, or its inflation to $15 \mathrm{~mm}$. The entire procedure was performed by insufflating the least amount of $\mathrm{CO}_{2}$ needed to maintain visualization of the biliary tree. All procedures were performed by a single experienced endoscopist ( $>1,000$ previous ERCP procedures) assisted by an expert nurse. Prior to the completion of this study, the endoscopist had performed several DPC procedures using an ultrathin endoscope assisted by a guidewire or overtube.

Patients gave written consent for ERCP and cholangioscopy. The study was approved by the local IRB of the Hospital General Universitario de Elche (Alicante, Spain).

Statistical calculations were performed using the SPSS 17 program. All quantitative variables followed a normal distribution (Kolmogorov-Smirnov test), so that these variables are expressed as the mean, standard deviation and range. To compare quantitative variables the parametric Student $t$ test was used. A $p<0.05$ was considered significant. 
Table I. Patient characteristics and indications for cholangioscopy

\begin{tabular}{ll}
\hline Patients included & $\mathrm{n}=15$ \\
Age (yrs) & $77.7 \pm 10.8$ (45-91) \\
Sex (M/F) & $8 / 7$ \\
$\begin{array}{l}\text { Indication for cholangioscopy } \\
\text { Filling defects after removal of } \\
\text { choledocholithiasis }\end{array}$ & 9 \\
$\quad$ Electrohydraulic lithotripsy & 5 \\
Indeterminate biliary stricture & 1 \\
\hline Age is expressed as mean + standard deviation and range (in parentheses).
\end{tabular}

\section{RESULTS}

Between December 2012 and June 2013, ninety ERCP procedures were performed. A DPC was performed in 15 patients $(17 \%)$. The characteristics of the patients and the indications for cholangioscopy are shown in table I. Most cases had suspicion of retained stones after extracting all the choledocolitiasis by standard ERCP. Another group of patients was referred to electrohydraulic lithotripsy as they had previously one or more ERCP in which extraction of stones by several methods (including mechanical lithotripsy) was not possible. In one case the indication was the evaluation of indeterminate stenosis of the lower third of the bile duct diagnosed by abdominal CT and MRI cholangiography. These patients had undergone a prior ERCP in which biliary cytology was negative for malignancy.

The findings and results of cholangioscopy are shown in table II. In cases of suspected retained choledocholi- thiasis, stones were observed by cholangioscopy in 3/9 $(33.3 \%)$ cases. In all cases, the stones were extracted with the intraductal balloon. In patients referred for electrohydraulic lithotripsy fragmentation, complete removal of the lithiasis was achieved in four cases. In the remaining case, which had multiple large stones, fragmentation of the lithiasis was partial, so a second session of electrohydraulic lithotripsy (not included in the present study) was necessary. The patient with indeterminate bile duct stenosis had a lower third stenosis with regular contours. Biopsies were taken with a $2 \mathrm{~mm}$ biopsy forceps through the working channel of the ultrathin endoscope. The pathology report was negative for malignancy. Figure 1 shows various aspects of DPC with ultrathin endoscope.

Table III shows the main results of the study. Access into the common bile duct with the ultrathin endoscope and freehand technique was possible in $14 / 15$ cases. In one case the access was not possible. In this patient a retained choledocholithiasis was suspected after sphincterotomy and stone extraction. After 22 minutes trying to access the common bile duct, the endoscopist decided to end the procedure. In this case, the ultrathin endoscope slipped through the sphincterotomy area, so that it was not possible to get a stable position to enter the bile duct. Time to access to the common bile duct was significantly higher in patients who had undergone a prior sphincterotomy and/ or sphincteroplasty versus those with "naive" papillae in which sphincterotomy was carried out in the current procedure $(4.7 \pm 1.5 \mathrm{vs} .16 .9 \pm 11.2$ minutes, $\mathrm{p}=0.01)$.

In $13 / 15$ cases it was possible to examine the whole common bile duct to the confluence. In one case, the ultrathin endoscope could not be advanced to the confluence

Table II. Results of cholangioscopy

\begin{tabular}{llllll}
\hline & Age & Sex & Indication & Common bile duct diameter & Outcome \\
\hline 1 & 80 & Female & Retained stone suspicion & 12 & No lithiasis \\
2 & 75 & Male & Retained stone suspicion lithiasis & Bening stenosis \\
3 & 45 & Male & Indeterminate stenosis & 11 & Single 5 mm lithiasis \\
4 & 85 & Male & Retained stone suspicion & 12 & No lithiasis \\
5 & 74 & Male & Retained stone suspicion & 10 & No lithiasis \\
6 & 73 & Female & Retained stone suspicion & 10 & Complete extraction \\
7 & 78 & Female & Electrohydraulic lithotripsy & 18 & Single 6 mm lithiasis \\
8 & 91 & Female & Retained stone suspicion & 18 & Complete extraction \\
9 & 59 & Male & Electrohydraulic lithotripsy & 18 & Extracción parcial \\
10 & 79 & Male & Electrohydraulic lithotripsy & 18 & Complete extraction \\
11 & 88 & Male & Electrohydraulic lithotripsy & 15 & Complete extraction \\
12 & 74 & Female & Electrohydraulic lithotripsy & 15 & 5 lithiasis < 6 mm \\
13 & 80 & Female & Retained stone suspicion & 15 & No lithiasis \\
14 & 82 & Male & Retained stone suspicion & 15 & No lithiasis \\
15 & 73 & Female & Retained stone suspicion & &
\end{tabular}



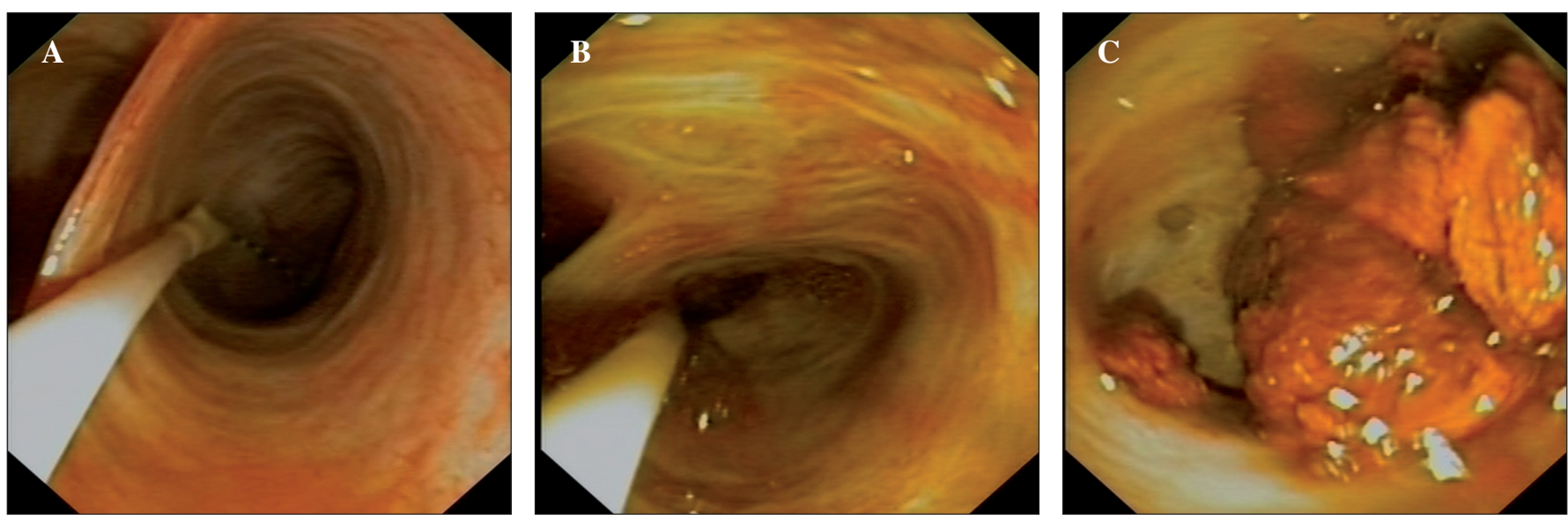

Fig. 1. Different aspects of cholangioscopy. A. Ultrathin endoscope with anchoring balloon. The output of a dilated cystic duct can be observed to the left. B. Visualization of the biliary confluence. The balloon catheter is anchored in a left intrahepatic branch. C. Choledocholithiasis.

because it was not possible to anchor the intraductal balloon in an intrahepatic branch of the biliary tree. The other case was the above mentioned in which access to the common bile duct was not achieved.

During the ERCP two complications were observed: One patient had a maintained oxigen desaturation $<85 \%$ during cholangioscopy. The ultrathin endoscope was removed, sedation stopped and the patient was ventilated without endotracheal intubation. After a few minutes she recovered completely. Another patient had sustained hypotension requiring ephedrine. No cases of significant bradycardia or tachycardia were observed during cholangioscopy. All patients were discharged within 24 hours except for one case.

This was a woman who had melena before ERCP. During ERCP the upper gastrointestinal tract was examined, with no evidence of potentially bleeding lesions. After ERCP she continued with melena and anaemia. Twentyfour hours after ERCP, an upper endoscopy was performed. No blood was found in the esophagus, stomach and duodenum. The area of the papilla was carefully inspected, without observing a cause of bleeding. Subsequently, melena disappeared and the patient was discharged five days after ERCP. One patient was readmitted 4 days after ERCP due to vomiting without abdominal pain. ERCP complications were discarded. The patient had not had a bowel movement since ERCP. Vomiting disappeared after administering an enema, which was effective, and the patient was discharged in 24 hours.

\section{DISCUSSION}

Cholangioscopy is a useful technique for the study and treatment of biliary disease. For many years "mother-baby" systems have been used for this technique. However, these systems are not widely used as they require two skilled endoscopists. In addition, the "baby" scope is very fragile. In recent years there has been renewed interest in cholangioscopy with the emergence of new systems, as SpyGlass $^{\circledR}(2)$ and Polyscope ${ }^{\circledR}(3)$ requiring only one operator. However, these systems, as they are designed today, have some drawbacks. Its image quality is not optimal and the working channel is too small. Therefore research is ongoing in the development of new systems.

One system that has attracted more attention in recent years is the use of ultrathin endoscopes to gain access directly to the bile duct. This has been called "direct peroral cholangioscopy" (DPC). Ultrathin endoscopes are widely available, endoscopists are familiar with them, and its image quality is optimal thanks to a high-resolution CCD chip (with the possibility of virtual chromoendoscopy). Also, they are not as fragile as the "mother- baby". Finally, they have a working channel of $2 \mathrm{~mm}$. However, they have two main disadvantages: First, access to the bile duct can be difficult at first. Therefore it has been pro-

Table III. Main results

\begin{tabular}{ll}
\hline Common bile duct diameter $(\mathrm{mm})$ & $13.9 \pm 3.1(10-18)$ \\
Access to common bile duct & $13 / 14(92.9 \%)$ \\
Time to access common bile duct $(\mathrm{min})^{*}$ & $10.4 \pm 9.7(3-40)$ \\
Biliary confluence visualization & $13 / 15(86.7 \%)$ \\
Time to confluence $(\mathrm{min})^{* *}$ & $13.3 \pm 8.5(2-30)$ \\
Total ERCP time $(\mathrm{min})^{* * *}$ & $81.7 \pm 21(57-126)$ \\
Complications & $2 / 15(13.3 \%)$ \\
\hline
\end{tabular}

Quantitative variables are expressed as mean \pm standard deviation and range (in parentheses)

*Time from insertion of the ultrathin endoscope through the mouth to enter the common bile duct. **Time from entry into common bile duct to position the tip of the ultrathin endoscope within $1 \mathrm{~cm}$ or less of the biliary confluence. $* * *$ Time from starting of ERCP, including cholangioscopy, until finishing the entire procedure. 
posed to insert these endoscopes into the bile duct over a previously placed guidewire. This technique can be cumbersome. The second disadvantage is that, if the full examination of the common bile duct is intended, it is not always possible to access the upper portion of it. This is due mainly to stomach and duodenum loops that provoke slippage the endoscope out of the bile duct.

To solve this problem, several support systems have been proposed, such as the use of enteroscopy overtube (5), intraduodenal balloon in parallel with the ultrathin endoscope (6), intraductal anchor systems with a special guidewire including Kautz guidewire (7) and the intraductal anchor with a balloon $(8,9)$. The latter system consists of a Fogarty balloon passed through the working channel of the ultrathin endoscope. The balloon is pushed up to an intrahepatic branch where it is inflated to get your anchor at this level. The ultrathin endoscope is advanced over the balloon to examine the common bile duct. In a previous study, two techniques for examination of the common bile duct were compared: A rigid guidewire vs. intraductal balloon. This study showed a higher success rate to examine the full common bile duct with the latter (45 vs. $95 \%$ ) (8). Recently, a special balloon was designed which was passed through the duodenoscope and anchored into an intrahepatic branch. The duodenoscope could be removed and the inflated balloon left in place. The ultrathin endoscope could be introduced over the balloon, achieving an access rate to the common bile duct of $100 \%$ (9). However, the manufacturer decided to remove the balloon from the market after a severe complication was reported (10). In all these studies, biliary access was performed leaving a guidewire inside the bile duct, after which the duodenoscope was removed and the ultrathin endoscope was inserted over the guidewire.

In the present study we aimed to find some way to make DPC with an ultrathin endoscope easier. First, we used a freehand technique, i.e., accessing the bile duct without the need of a guidewire. Also, only commercially available material was used.

We found that cholangioscopy is a useful technique in the assessment of residual stones as well as in the management of difficult stones. The percentage of indications of cholangioscopy in our study is high (17\% of all ERCP), due to a large number of indications for suspected retained choledocholithiasis. In fact, ever since cholangioscopy has been available in our institution, we have had a low threshold for performing cholangioscopy when there is the slightest doubt that a stone can be retained. Thus, retained stones could be detected in $33 \%$ of cases with negative cholangiogram, which would otherwise have been left within the bile duct. This percentage is similar to that published in previous studies (11). For patients referred for electrohydraulic lithotripsy, clearing of choledocholitiasis was achieved in $80 \%$ of cases in a single session. Also, we confirmed the safety of DPC with an ultrathin endoscope. Only two complications, probably not related to DPC but to the sedoanalgesia, were observed.
We have observed a high success rate when accessing the common bile duct with the freehand technique, similar to the rate observed in studies in which access was gained over a guidewire (8). One of the interesting findings of our study is that the access time is substantially shortened if a sphincterotomy and/or sphincteroplasty had been performed in a previous ERCP, but time is greatly increased if we try to access after a sphincterotomy performed during the ongoing procedure. In our opinion, this could be explained because after sphincterotomy, oedema of the papilla occurs. Thus, especially if the sphincterotomy is not very wide, it may be difficult to locate the papilla orifice, which is frequently collapsed. As a result, it can be difficult to pass the ultrathin endoscope through the papilla into the bile duct.

Regarding the full examination of the common bile duct, the results of our study are similar to those previously reported in which an intraductal balloon was used. Thus, in the study by Parsi et al. (9) the common bile duct could be fully examined in $93 \%$ of cases. Only in one case, a patient with a "sigmoid" shaped common bile duct, full examination was not achieved. In the study by Moon et al. (8), a full examination of the common bile duct was possible in $95.2 \%$ of cases. Only in one case it was not possible because the intraductal balloon could not be anchored. In our series, in two cases full examination of the common bile duct was not possible. In one patient, it was not possible to access the bile duct. In another patient, the anchoring of intraductal balloon failed. One must keep in mind that our study was designed to assess the proportion of complete examination of the common bile duct, but in clinical practice it is not always necessary to examine the common bile duct to the confluence. In many cases simply accessing the lower third of the bile duct will be sufficient to achieve our goal. If a retained stone us suspected, we can irrigate and aspirate through the working channel of the ultrathin endoscope which leads to mobilization of the stones even if they are high in the intrahepatic branches. For therapeutic manoeuvres, such as intraductal lithotripsy, it may be sufficient to place the ultrathin endoscope in the lower third of the bile duct.

One aspect that we should pay special attention to are the complications related to the procedure. As previously mentioned, a serious complication related to the intraductal balloon has been communicated (10). In this case, an air embolism with systemic residual hemiplegia occurred in a patient with right-left heart communication. If no rightleft cardiac or pulmonary communication exists there is no risk of systemic embolism. The risk of air embolism could be related to the insuflation of ambient air into the bile duct as the endoscope itself may block the air outlet into the duodenum. In another study a case of biloma following the procedure described (9), with resolution after antibiotic treatment and placing a biliary stent. One must keep in mind, therefore, that injury can be caused to the intrahepatic bile ducts due to the anchor of the balloon. 
The limitations of the present study are the small number of patients included. In addition, the common bile duct was dilated in all patients (no case had a common bile duct less than $10 \mathrm{~mm}$ ), so the results may not be reproducible in cases where the calibre of the common bile duct is less. Moreover, all examinations were performed in the setting of a university hospital by a single endoscopist with broad experience in ERCP and some previous experience in DPC, i.e. not starting from zero at the time of the study. The results cannot be extrapolated to other circumstances. In this regard, we must say that the DPC is not an easy technique. The learning curve has not been defined. Also, it can be accompanied by serious complications, so for the moment, it seems reasonable to recommend that it only be performed by endoscopists with broad experience in ERCP. Another limitation of the present study is that the monitoring of patients was short, only 24 hours. After 24 hours, complications were recorded by reviewing the computerized history at day 30 after ERCP. This may cause the number of complications to be underestimated.

Undoubtedly the DPC technique with an ultrathin endoscope is a major advance in the way we approach ERCP, its strengths being the excellent image quality and the possibility of therapeutic manoeuvres through a working channel of $2 \mathrm{~mm}$. However, the ultrathin endoscopes are not specifically designed for access to the biliary tree. Technical improvements are needed if we want direct peroral cholangioscopy to become a standard in the examination of the bile duct. In this sense, prototypes are been developed that will undoubtedly lead to a technical improvement in this field (12).

In conclusion, the DPC with ultrathin endoscope can be done with freehand technique. With the aid of an anchoring intraductal balloon, full examination of the common bile duct can be achieved in a high percentage of cases.

\section{REFERENCES}

1. Fukuda Y, Tsuyuguchi T, Sakai Y, Tsuchiya S, Saisyo H. Diagnostic utility of peroral cholangioscopy for various bile-duct lesions. Gastrointest Endosc 2005;62:374-82.

2. Chen YK, Pleskow DK. SpyGlass single-operator peroral cholangiopancreatoscopy system for the diagnosis and therapy of bile-duct disorders: a clinical feasibility study (with video). Gastrointest Endosc 2007;65:832-41.

3. Cennamo V, Luigiano C, Fabbri C, Maimone A, Bazzoli F, Ceroni $\mathrm{L}$, et al. Cholangioscopy using a new type of cholangioscope for the diagnosis of biliary tract disease: A case series. Endoscopy 2012;44:878-81.

4. Larghi A, Waxman I. Endoscopic direct cholangioscopy by using an ultra-slim upper endoscope: A feasibility study. Gastrointest Endosc 2006;63:853-7.

5. Choi HJ, Moon JH, Ko BM. Overtube-balloon-assisted direct peroral cholangioscopy by using an ultra-slim upper endoscope (with videos) Gastrointest Endosc 2009;69:935-40.

6. Mori A, Ohashi N, Nozaki M, Yoshida A. Feasibility of duodenal balloon-assisted direct cholangioscopy with an ultrathin upper endoscope. Endoscopy 2012;44:1037-44.

7. Beyna T, Lenze F, Hengst K, Ullerich $\mathrm{H}$. A new anchoring technique for accessing the bile duct during direct peroral cholangioscopy using the guide probe of Kautz. Endoscopy 2012;44(Supl. 2) UCTN:E372-3.

8. Moon JH, Ko BM, Choi HJ, Hong SJ, Cheon YK, Cho YD et al. Intraductal balloon-guided direct peroral cholangioscopy with an ultraslim upper endoscope (with videos). Gastrointest Endosc 2009;70:297-302.

9. Parsi MA, Stevens T, Vargo JJ. Diagnostic and therapeutic direct peroral cholangioscopy using an intraductal anchoring balloon. World J Gastroenterol 2012;18:3992-6.

10. Efthymiou M, Raftopoulos S, Antonio Chirinos J, May GR. Air embolism complicated by left hemiparesis after direct cholangioscopy with an intraductal balloon anchoring system. Gastrointest Endosc 2012;75:221-3

11. Lee YN, Moon JH, Choi HJ Min SK, Kim HI, Lee TH, et al. Direct peroral cholangioscopy using an ultraslim upper endoscope for management of residual stones after mechanical lithotripsy for retained common bile duct stones. Endoscopy 2012;44:819-24.

12. Itoi T, Reddy DN, Sofuni A, Ramchandani M, Itokawa F, Gupta R, et al. Clinical evaluation of a prototype multi-bending peroral direct cholangioscope. Dig Endosc 2014;26:100-7. 